\title{
Adsorption of bilayers and multilayers of cationic and anionic co-polymers of acrylamide on silicon oxide
}

\author{
Lars Wågberg, ${ }^{\mathrm{a}, *}$ Gunilla Pettersson, ${ }^{\mathrm{b}}$ and Shannon Notley ${ }^{\mathrm{b}}$ \\ a Fibre Technology, KTH, Stockolm, Sweden \\ ${ }^{\mathrm{b}}$ Natural Environmental Sciences, Midsweden University, Sundsvall, Sweden
}

Received 18 August 2003; accepted 21 November 2003

Available online 22 January 2004

\begin{abstract}
Silica surfaces were consecutively treated with copolymers of cationic and anionic polyacrylamides (C-PAM and A-PAM, respectively) and the layer-by-layer build-up was continuously monitored with the aid of stagnation point adsorption reflectometry (SPAR). Four different charge densities of the cationic polymer and one charge density of the anionic polymer were studied. The solid substrate used in the investigation was an oxidized Si wafer, the charge of which was varied by performing the measurements at different $\mathrm{pH}$. Adsorption measurements were performed both in deionized water and with a background electrolyte concentration of $0.01 \mathrm{M} \mathrm{NaCl}$. The results show that the adsorption of C-PAM at pH 6 was dominated by electrostatic interactions. However, a significant nonionic contribution to the adsorption of C-PAM on $\mathrm{SiO}_{2}$ was detected-when the results of adsorption measurements conducted in deionized water and in $0.01 \mathrm{M} \mathrm{NaCl}$ were compared. At $\mathrm{pH}$ 9, the adsorption of C-PAM onto $\mathrm{SiO}_{2}$ was found to be geometrically restricted since the adsorption stoichiometry between the polymer charges and the charges on the surface was less than 1 irrespective of the charge of the C-PAM. Adsorption of the A-PAM onto the C-PAM covered surface increases as a function of the adsorbed charges in the first layer. Experiments showed that it was possible to form multilayers of polyelectrolytes on the $\mathrm{SiO}_{2}$ surface provided the charge of the C-PAM was high enough. The critical charge of the polyelectrolyte for the formation of multilayers was also dependent on the charge of the substrate; that is, the lower the surface charge the higher the critical charge of the C-PAM. The substrate affected the amount of polyelectrolyte adsorbed up to the fifth layer. For further layers there was almost a stoichiometric relationship between the charges of the polyelectrolytes in consecutive layers. Results from studies of the formed multilayers with a quartz crystal microbalance (QCM-D) indicated that there was a close correlation between energy dissipation into the multilayers and a decrease in the adsorption as detected with SPAR. This in turn indicates that a decrease in the reflectometer signal does not necessarily indicate a decrease in adsorption.
\end{abstract}

(c) 2003 Elsevier Inc. All rights reserved.

\section{Introduction}

Polyelectrolytes are used in many industrial applications, as dispersing agents and as flocculants, as wet and dry strength agents in the paper industry, and also in waste water treatment, paint, and cosmetics. In most of these applications it is essential that the polyelectrolytes are adsorbed onto the solid substrates to be flocculated, dispersed, or strengthened. Great effort has been devoted over the years to theoretically describing the adsorption of polyelectrolytes due, in large part, to this industrial relevance [1-5]. Furthermore, the influence of the polyelectrolytes on the stability of dif-

\footnotetext{
* Corresponding author.

E-mail address: wagberg@pmt.kth.se (L. Wågberg).
}

ferent types of colloidal dispersions has been extensively studied [6,7].

The interest in polyelectrolyte adsorption has recently increased due to the development of the concept of multilayer adsorption of polyelectrolytes onto solid substrates [8-12]. A basis for the preparation of multilayer thin films on solid substrates is the overcompensation and charge reversal of the substrate due to adsorption of an oppositely charged polymer. In self-consistent field (SCF) lattice theories as described in $[1,13,14]$, recharging of the surfaces can only occur if there is also a nonionic interaction between the polymer segments and the surface. The recharging of the surfaces, as found in many investigations of polyelectrolyte adsorption at the solid-liquid interface, is hence often referred to as a nonequilibrium effect $[15,16]$ for the case where nonionic interactions are absent. According to re- 
cent theories of polyelectrolyte adsorption on oppositely charged surfaces [3-5], recharging of the surface can be achieved only under certain conditions. A theoretical extension to polyelectrolyte multilayer formation has also been made [3,4]. Recently a new approach based on site-specific adsorption of polyelectrolytes was also developed to describe both monolayer and multilayer adsorption of polyelectrolytes [17]. However, despite these developments, it must still be concluded that a full theoretical description of polyelectrolyte multilayer (PEM) formation and its dependence on surface properties of the substrate, concentration of low-molecular-mass electrolytes, and solution properties of the polyelectrolytes is still not available. Furthermore, the currently available theories describing PEM formation can only be used to describe multilayers having linear growth, whereas multilayers with exponential growth, i.e., typical for most of the multilayers described in this report, cannot be described with current theories. The molecular mechanisms behind the difference between linear and exponential growth of PEM has been recently reported [18].

In practice the formation of PEM is achieved by saturating a surface with an oppositely charged polymer, rinsing with the solvent, and subsequent treatment with a polymer of charge opposite to that of the first polymer. This procedure is then repeated for the formation of an increasing number of layers, and as previously demonstrated [19,20], the treated surfaces are recharged by exposure to the different polyelectrolytes. The formation of the PEM is influenced by the substrate for the first few layers, and following this there is a stoichiometric compensation of the charges between the polyelectrolytes for the main part of the PEM. Only the outer layers of the PEM carry the net charge of the multilayer [21]. Nonequilibrium effects will also influence the formation of the PEM. It has been demonstrated both theoretically and experimentally that the adsorption of a polyelectrolyte onto an oppositely charged surface would likely form a barrier against further adsorption even before surface charge compensation. This is due mainly to the slow reconformation of the polyelectrolyte on the surface of the substrate $[15,16]$. This was also shown for the adsorption of an anionic polyacrylamide onto cellulose fibers that had been treated with a cationic copolymer of polyacrylamide [22]. By measuring the adsorption at different delay times after adsorption of the cationic polyacrylamide, it was found that the subsequent adsorption decreased with increasing delay time. This was taken as indirect evidence of the existence of a temporary recharged surface.

With this as a background, the purpose of the present work was to clarify how the adsorption of anionic and cationic copolymers of acrylamide in bilayers and multilayers was affected by the charge of the surface $\left(\mathrm{SiO}_{2}\right)$, the degree of substitution of a cationic copolymer, and the concentration of electrolyte in the solution. From these results it should be possible to determine critical limits for the buildup of multilayers and to clarify if the build-up of bilayers follows the trends predicted by the different adsorption theories.

\section{Experimental}

\subsection{Methods}

In order to monitor the adsorption of polyelectrolytes a stagnation point adsorption reflectometer (SPAR) from the Agricultural University of Wageningen, The Netherlands, was used according to methods described in $[23,25]$. In this method laser light is reflected off a smooth surface and the change in intensity of the parallel, $I_{p}$, and perpendicular, $I_{s}$, components of the incoming laser light is measured. The ratio $S=\left(I_{s}-I_{p}\right) /\left(I_{s}+I_{p}\right)$ is then calculated. The change in this ratio with adsorption of, for example, surfactants and polymers was shown to be proportional to the amount adsorbed [24]. Data from the SPAR is presented as the ratio $\Delta S / S_{0}$, where $\Delta S$ is the change in the $S$-value upon adsorption and $S_{0}$ is the initial $S$ value before adsorption. The $\Delta S$ value was then converted to the adsorbed amount (or surface excess) according to a procedure previously described $[24,25]$ using a simplified evaluation procedure according to the equation

$\Gamma=\frac{\Delta S}{S_{0}} \frac{1}{0.129(d n / d c)}$,

where $d n / d c$ is the refractive index increment for the polymer system used in the adsorption measurements and $0.129(d n / d c)$ is a simplified expression for the $A_{s}$ (sensitivity factor) defined in [23].

As remarked in connection with the adsorption results later in this paper, this simplified procedure correlates very well with the four-layer optical evaluation procedure for the results from SPAR measurements [23]. In all adsorption measurements silicon wafers from MEMC Electronic Materials, SpA, Italy $(150 \mathrm{~mm} / \mathrm{CZ} / 1-0-0 /$ Arsenic/N + Type/Resis 0.001-150.000), were used. The Si wafers were oxidized by heating them at $1000^{\circ} \mathrm{C}$ for $30 \mathrm{~min}$ in dry air in order to produce $\mathrm{a} \mathrm{SiO}_{2}$ layer with a defined thickness on the surface. Great care was taken to oxidize and use the surfaces with exactly the same storage time between the oxidation and adsorption measurements and the surfaces were stored under dust-free conditions in a desiccator between oxidation and adsorption measurements.

In the SPAR, a stagnation point flow setup was used. In this way a well-defined hydrodynamic situation is created where diffusion of polymers to the surface is the only transport mechanism.

For the adsorption measurements, the oxidized silicon wafer was mounted in the SPAR equipment and the solvent flow started. A stable baseline was established before the solvent flow was replaced with a polymer solution of known concentration. The flow of polymer was continued until a stable reading of the $S$ signal was achieved, i.e., until there was a plateau in the adsorbed amount. Following 
this the surface was again exposed to the solvent in order to rinse the cell and to remove any nonadsorbed polymer. A solution with a polymer of charge opposite to the previously added polymer was introduced into the cell. A new rinse with the solvent was then used before a solution of the first polymer was reintroduced into the cell. This procedure was then repeated several times in order to investigate if it was possible to build multilayers with cationic copolymers with different charge densities and an anionic copolymer. The measurements were conducted both at $\mathrm{pH} 6$ and at $\mathrm{pH} 9$ in deionized water and in $0.01 \mathrm{M} \mathrm{NaCl}$. The repeatability and reproducibility of the SPAR measurements were excellent and the coefficient of variation of the adsorption values was less than $8 \%$. The build-up of the polyelectrolyte multilayers was also studied with the aid of a quartz crystal microbalance with dissipation (QCM-D equipped with an axial flow chamber from Q-Sense AB, Göteborg, Sweden). QCM-D allows the simultaneous measurements of both frequency changes and energy dissipation changes during the build-up of the multilayers. The sensor crystals used in the measurements were 5-MHz AT-cut crystals coated with $\mathrm{SiO}_{2}$ (Q-Sense AB). All measurements were recorded at four different frequencies $(5,15,26,36 \mathrm{MHz})$, but the third overtone $(15 \mathrm{MHz})$ has primarily been used in the evaluation of the data due to its stability. In the experiments with the QCM-D equipment, the same procedure as used in the SPAR equipment was used for construction of the polyelectrolyte bilayers and multilayers. The QCM-D liquid chamber was temperature-stabilized to $+20 \pm 0.1^{\circ} \mathrm{C}$ during the measurements and experiments were conducted only with one of the cationic polyacrylamides, the polymer with a $\mathrm{DS}=0.14$ $(\mathrm{mol} \%)$ and at $\mathrm{pH} 9$ and with a $\mathrm{NaCl}$ concentration of $0.01 \mathrm{M}$.

Polyelectrolyte titration [26] was used to characterize the charge of the polyelectrolytes used. An automatic titration unit similar to the equipment described in [27] was used to determine the end point in the titration curves.

\subsection{Materials}

The cationic copolymers used in this investigation were copolymers of acrylamide and amino-propyl-trimethyl-ammoniumchloride (APTAC). Four different cationic copolymers were investigated and the properties of these polymers are summarized in Table 1. The anionic polymer was a copolymer of acrylamide and acrylic acid and the molar degree of substitution of anionic groups was 16\% (according to the manufacturer), which resulted in a calculated charge of $1.86 \mathrm{meq} / \mathrm{g}$. The charge of the cationic copolymers, as determined by polyelectrolyte titration, is given in Table 1 . Included in Table 1 are the degree of substitution (DS) of cationic groups as calculated from these charge measurements and also the $d n / d c$ values of the polymers needed to convert the $\Delta S / S_{0}$ values to an adsorbed amount in $\mathrm{mg} / \mathrm{m}^{2}$ according to an earlier described procedure [25].
Table 1

Summary of the characteristics of the polyelectrolyte used in this investigation

\begin{tabular}{lccccr}
\hline Material & $\begin{array}{c}\text { Charge } \\
(\mathrm{meq} / \mathrm{g})\end{array}$ & $\begin{array}{c}\mathrm{DS} \\
(\%)\end{array}$ & $\begin{array}{c}M_{w} \\
\times 10^{-6}\end{array}$ & $\begin{array}{c}d n / d c \\
(\mathrm{ml} / \mathrm{g})^{\mathrm{a}}\end{array}$ & $\begin{array}{r}\text { Intr. visc. } \\
(\mathrm{dl} / \mathrm{g})^{\mathrm{b}}\end{array}$ \\
\hline C-PAM 1 & 0.58 & 4.5 & & 0.1732 & 9.1 \\
C-PAM 2 & 1.02 & 8.5 & & 0.1710 & 9.7 \\
C-PAM 3 & 1.54 & 14 & $10^{\mathrm{c}}$ & 0.1665 & 10.4 \\
C-PAM 4 & 2.57 & 28 & & 0.1625 & 6.5 \\
A-PAM & 1.86 & 16 & $5^{\mathrm{d}}$ & 0.1720 & 14.0 \\
\hline
\end{tabular}

a These values were taken from [28] for the C-PAM and from [29] for the A-PAM

b Measured in $1 \mathrm{M}$ solution of $\mathrm{NaCl}(77 \%)$ and $\mathrm{NaH}_{2} \mathrm{PO}_{4}(21 \%)$.

c As determined from static light scattering in $1 \mathrm{M} \mathrm{NaCl}$.

d According to the manufacturer.

The C-PAM polyelectrolytes had similar intrinsic viscosities at $1 \mathrm{M} \mathrm{NaCl}$ and therefore only one polymer was subjected to static light scattering to determine the molecular weight. All polymers were specially prepared and kindly supplied by Ciba Specialty Chemicals (Allied Colloids, Bradford, UK). Since the manufacturer carefully washed the polymers with repeated precipitation in acetone from water, they were used without further purification. The polymers were dissolved in deionised water under gentle stirring for $16 \mathrm{~h}$ with the aid of a magnetic stirrer prior to use and the desired $\mathrm{pH}$ and salt concentrations of the polymer solutions were adjusted subsequent to dissolution. Fresh polymer solutions were prepared every day. $\mathrm{NaCl}$ used in the investigation was of analytical grade and supplied by Kebo AB, Stockholm, Sweden and the water used was Milli-Q water where all electrolytes and organic contaminants had been removed.

\section{Results and discussion}

\subsection{Adsorption of C-PAM and bilayers of C-PAM and A-PAM onto $\mathrm{SiO}_{2}$}

In the initial experiments C-PAM with different charge densities were adsorbed onto $\mathrm{SiO}_{2}$ surfaces at different salt concentrations and at different $\mathrm{pH}$. The results from measurements at $\mathrm{pH} 9$ and at a salt concentration of $0.01 \mathrm{M}$ $\mathrm{NaCl}$ are summarized in Figs. $1 \mathrm{a}$ and $1 \mathrm{~b}$. The $\Delta S / S_{0}$ results, collected from the reflectometer measurements, were then converted to adsorbed amounts, in $\mathrm{mg} / \mathrm{m}^{2}$, according to an earlier published procedure [25]. This is a simplified procedure compared to the procedure described in [23] but was chosen due to the lack of exact knowledge of the oxide layer thickness on the wafers. However, recent results where this procedure was compared with a more rigorous measurement of the adsorption by ellipsometry [30] for one of the used polymers, C-PAM 3, showed an excellent agreement between the methods and therefore this way of data treatment was justified. The adsorbed amount measured as $\mathrm{mg} / \mathrm{m}^{2}$ and $\mu \mathrm{eq} / \mathrm{m}^{2}$ is shown in Figs. 1a and $1 \mathrm{~b}$. The polyelectrolyte 


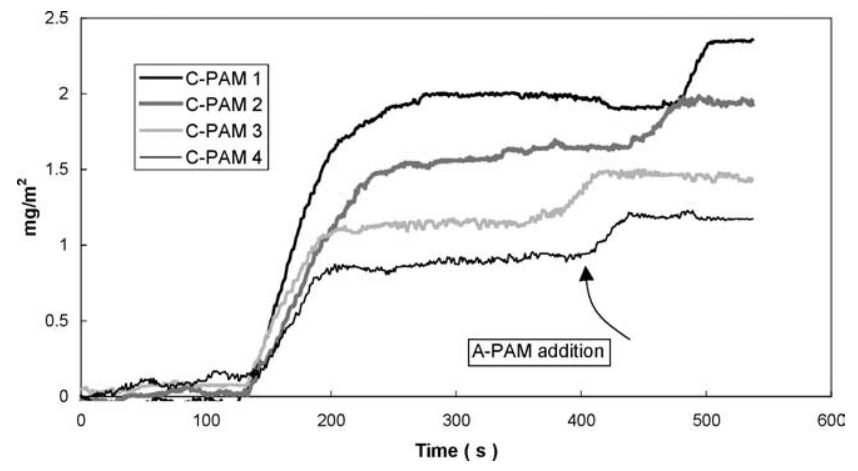

(a)

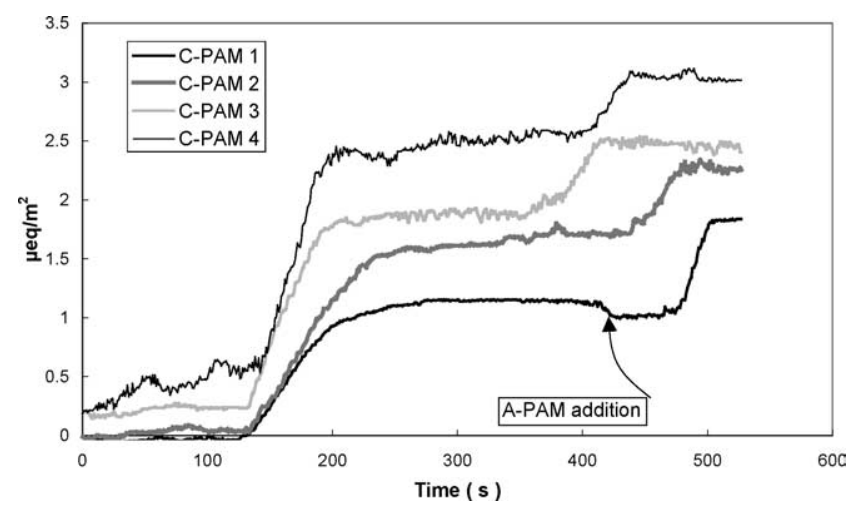

(b)

Fig. 1. Adsorption in (a) $\mathrm{mg} / \mathrm{m}^{2}$ and (b) $\mu \mathrm{eq} / \mathrm{m}^{2}$ of C-PAM and A-PAM to $\mathrm{SiO}_{2}$ surfaces at $0.01 \mathrm{M} \mathrm{NaCl}$, and $\mathrm{pH}$ 9. C-PAM 1 to C-PAM 4 correspond to the different charges of the C-PAM co-polymer. A summary of the characteristics of the polymers is given in Table 1.

charge was used to recalculate the adsorbed amount in grams to $\mu$ eq.

Figs. $1 \mathrm{a}$ and $1 \mathrm{~b}$ show that the adsorption in $\mathrm{mg} / \mathrm{m}^{2}$ increases with decreasing charge density, whereas the adsorption in $\mu \mathrm{eq} / \mathrm{m}^{2}$ decrease with increasing charge density. According to the SCF theory [1], for pure electrosorption the amounts of adsorbed charges should be the same for the different polymers. As is shown in Fig. 1b, this is obviously not the case, and there might naturally be several reasons for this. In order to investigate the controlling mechanisms behind the adsorption of the C-PAM on the silicon oxide surface, the adsorption was determined for two different $\mathrm{pH}$ conditions and at two different salt concentrations. The results from these adsorption measurements are summarized in Fig. 2 as saturation adsorption as a function of polyelectrolyte charge.

As can be seen in this figure, the adsorption measured as $\mathrm{mg} / \mathrm{m}^{2}$ increases as the salt concentration and the $\mathrm{pH}$ increase. The increase in adsorption with increasing salt concentration indicates that there is a nonelectrostatic interaction between the silica surface and the C-PAM contributing to the adsorption free energy for the C-PAM on the silica surface. There is a deviation for the polymer with the lowest charge density at pH 6; this will be discussed further later in this report. The increase in adsorption at higher $\mathrm{pH}$ is due

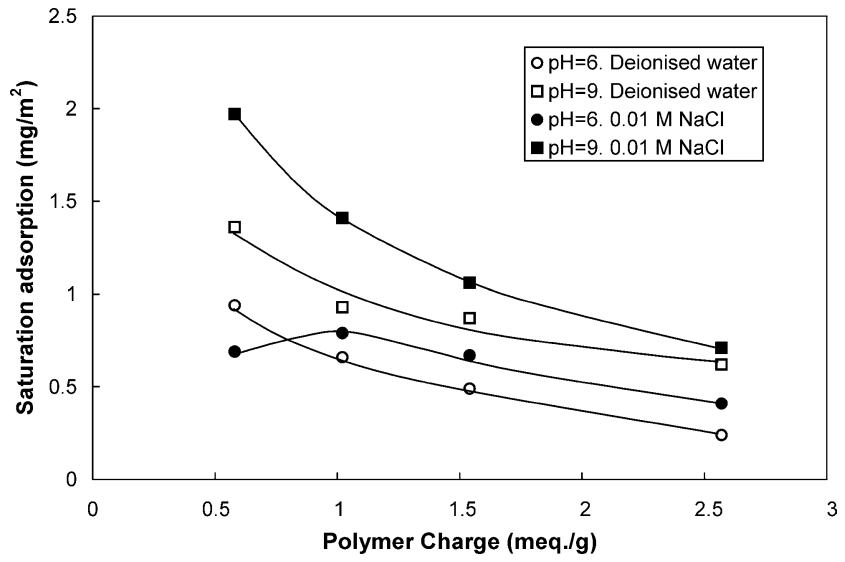

Fig. 2. Saturation adsorption for the C-PAM polyelectrolytes on the $\mathrm{SiO}_{2}$ surfaces at different $\mathrm{pHs}$ and salt concentrations.

to an increase in charge of the silica surface. Furthermore, it can also be seen that the absolute increase in adsorption at $\mathrm{pH}$ 9, at increased salt concentrations, is greater than that of pH 6. This is probably caused by a larger influence of the repulsion between the C-PAM due to the higher concentration of C-PAM on the surface at $\mathrm{pH} 9$.

However, in order to evaluate the relative influence of the interactions due to the charges on the polyelectrolyte and the charges on the surface, it was necessary to estimate the ratio between the charges of the surface and of the polyelectrolyte. The charges on the polyelectrolytes are given in Table 1 and the amount of adsorbed polymer charges was calculated from the adsorbed amount. In order to compare these results with the charges of the surface, the amount of adsorbed charge was divided by the charge of the $\mathrm{SiO}_{2}$ surface, as calculated according to the relationship introduced by Schindler [31], to give an overall adsorption stoichiometry between the polyelectrolyte and the surface,

$\log \left(\mathrm{OH}^{-}\right)=5.2 \log (\mathrm{pH})-4.78$,

where $\left(\mathrm{OH}^{-}\right)$is the number of charges per $\mathrm{nm}^{2}$.

The results from these calculations are shown in Fig. 3, where the charge ratio between the polyelectrolyte and the surface is presented as a function of the polyelectrolyte charge.

As can be seen in Fig. 3, it is possible to overcompensate the surface charges significantly at $\mathrm{pH} 6$, whereas it is not possible to overcompensate the surface charges at $\mathrm{pH} 9$ regardless of the salt concentration. If the adsorption at $\mathrm{pH} 6$ is first analyzed, together with the results shown in Fig. 2, these results also clearly show that there is a nonionic interaction between the polyelectrolyte and the surface, since the adsorption is increased upon salt addition [1]. This is also in accordance with earlier results [32] where it was shown that charge ratios of around 5 could be found for adsorption of cationic C-PAM onto silica surfaces. The increases in adsorption and adsorption stoichiometry can also be caused by the increase in surface charge following the salt addition but according to the data presented in [32], this large change in 


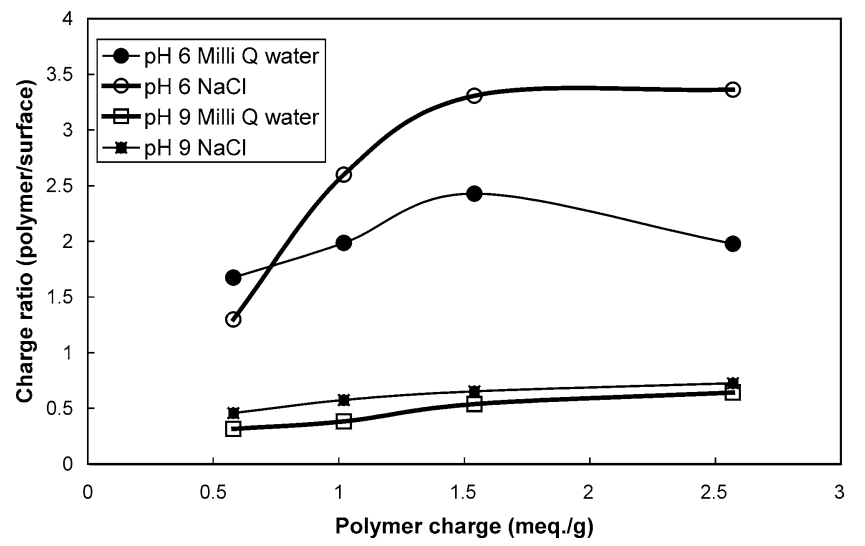

Fig. 3. Adsorption stoichiometry, i.e., the ratio between adsorbed polymer charges at saturation adsorption surface charge of the $\mathrm{SiO}_{2}$ surface calculated according to [31], as a function of the polyelectrolyte charge. The salt concentration was $0.01 \mathrm{M} \mathrm{NaCl}$ for the experiments where salt was added.

adsorption detected upon salt addition cannot be explained by a change in surface charge.

There is one experimental point deviating from this discussion regarding the increase in adsorption and charge ratio with increased salt concentration at $\mathrm{pH} 6$, and this is for the polyelectrolyte with the lowest charge density. For this polyelectrolyte there is a decrease in adsorption and charge ratio upon salt addition. The exact reason for this is not known but it must be mentioned that it is in accordance with the scaling theory for polyelectrolyte adsorption, as shown in [3]. This could indicate that the adsorption and surface recharging are controlled by factors other than the balance between electrosorption and nonionic interactions between the polyelectrolyte and the surface, as outlined in [1]. In this respect the present results stimulate more research regarding adsorption of low-charged polyelectrolytes on countercharged surfaces, similarly to the experiments shown here. It should also be mentioned that a decrease in adsorption at higher polyelectrolyte charge densities is expected, since "pure" electrosorption will be dominant for this type of polymer. This in turn leads to a decrease in overcharging of the surfaces, according to the SCF theory [1].

At $\mathrm{pH} 9$ a totally different situation is detected. First of all, the effect of the salt addition on the charge ratio is small and there is only a small, but significant, increase in charge ratio when the charge of the polyelectrolyte is increased. Second, the charge ratio is less than 1 indicating that it is not possible to neutralize all the charges on the surface at this $\mathrm{pH}$. However, the results in Figs. 1a and $1 \mathrm{~b}$ clearly show that it is possible to adsorb A-PAM onto a presaturated layer of C-PAM at $\mathrm{pH}$ 9. As pointed out by Netz and Joanny [5], the adsorption can be controlled either by charge compensation or by a geometrical saturation of the adsorbed layer. For the situation with geometrical saturation the polymer totally covers the surface and gives an extended adsorbed layer that will create a barrier against further adsorption, despite the fact that all charges on the surface have not been saturated. To test this hypothesis the adsorbed amount was recalculated as a surface coverage in $\mathrm{m}^{2} / \mathrm{m}^{2}$ by assuming that the polyelectrolyte maintains its solution conformation when adsorbed onto the surface. To do this, the $R_{g}$ (radius of gyration) was calculated from the composition of the polyelectrolyte and the results presented earlier by Audebert and co-workers [33] concerning the relationship between $M_{w}$ and $R_{g}$ for these types of polyelectrolytes. The adsorption values in Fig. 1a were used for this calculation and for $\mathrm{C}$ PAM 1 adsorbed at $\mathrm{pH}$ 9. In $0.01 \mathrm{M} \mathrm{NaCl}$, the surface coverage of the polyelectrolyte will be around $12 \mathrm{~m}^{2} / \mathrm{m}^{2}$. For C-PAM 3, the corresponding value is $8.5 \mathrm{~m}^{2} / \mathrm{m}^{2}$. This is a clear indication that the surface will be totally covered with polyelectrolytes at $\mathrm{pH} 9$ and that the surfaces can be recharged, provided that the thickness of the adsorbed layer is greater than the unperturbed thickness of the electrostatic double layer of the surface. In $0.01 \mathrm{M} \mathrm{NaCl}$ the double layer will have an extension of around $30 \AA$ and the $R_{g}$ for C-PAM 1 and C-PAM 3 is around 2000 and $1770 \AA$, respectively. Naturally the extension of the C-PAM layer from the surface at equilibrium will be lower than the $R_{g}$ value, but from these simple estimates it can be suggested that the surfaces are totally saturated at $\mathrm{pH} 9$ in the early stages of adsorption. Furthermore, this calculation suggests that unneutralized positive charges from the polyelectrolyte will dangle from the surface into the solution even though all the charges on the surface may not have been neutralized.

As an initial approximation it might seem reasonable to assume that the amount of A-PAM in the second layer should be a function of the charge ratio in the first adsorbed layer. In order to test this hypothesis, the adsorbed charges of A-PAM in the second layer were plotted as a function of the charge ratio, i.e., stoichiometry, in the first adsorbed layer and these results are shown in Fig. 4.

As can be seen in Fig. 4, there is a relationship between the charge ratio and the number of adsorbed A-PAM charges for the adsorption at $\mathrm{pH} 9$ and at $\mathrm{pH} \mathrm{6}$, at least for the results collected in the presence of $0.01 \mathrm{M} \mathrm{NaCl}$, even though there is a leveling off for higher charge ratios for the adsorption at $\mathrm{pH}$ 6. The reasons for this effect may be several and are discussed later in the section on the formation of

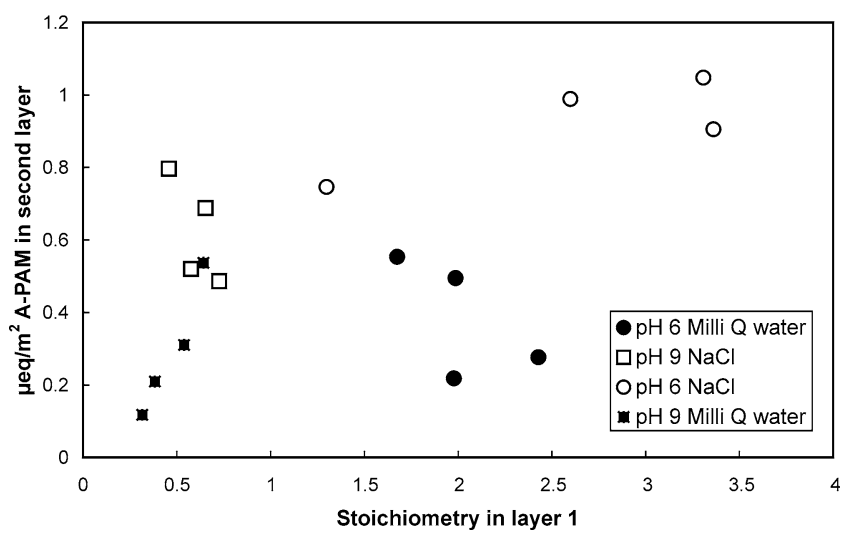

Fig. 4. Amount of adsorbed A-PAM charges as a function of the charge ratio, i.e., stoichiometry, in the first C-PAM layer. 
PEM of C-PAM and A-PAM. This result indicates that there is a correlation between the recharging of the $\mathrm{SiO}_{2}$ surface and the adsorbed amount. At $\mathrm{pH} 6$ this correlation is rather weak, especially when there is no added electrolyte, but at $\mathrm{pH} 9$ there is a clear correlation, indicating that the higher adsorbed amounts at this $\mathrm{pH}$ will result in a thicker adsorbed layer that will hence give a stronger interaction between the cationic and anionic polyelectrolytes. This, in turn, indicates that the surface properties influence the adsorption, at least in the second adsorbed layer, as has previously been observed for several other systems, as described in [12].

Fig. 4 also reveals that there is a much larger influence of the salt addition on the A-PAM adsorption at $\mathrm{pH} 6$ than at $\mathrm{pH}$ 9. This is most likely due to the significantly different structure of the adsorbed layer for different salt concentrations. This, in turn, will have a large effect on the adsorption of the A-PAM. As assumed in the theoretical model presented by Rubinstein and co-workers [3], the adsorbed layer might be divided into two different regimes, one inner region close to the surface with a rather constant concentration/density profile and one outer region with a parabolic density profile. The structure of these regions changes as the salt concentration is changed. It is possible that the accessibility of the A-PAM to the C-PAM charges changes significantly due to the increased screening by the added salt. The situation at higher $\mathrm{pH}$ is different since the adsorption in this case is geometrically restricted.

\subsection{Formation of PEM from C-PAM and A-PAM on $\mathrm{SiO}_{2}$}

A natural extension of the work concerning the formation of bilayers of C-PAM and A-PAM on the $\mathrm{SiO}_{2}$ was to investigate the possibility of forming multilayers with these polyelectrolytes on the $\mathrm{SiO}_{2}$ surfaces. Results from measurements at $\mathrm{pH} 9$ and $0.01 \mathrm{M} \mathrm{NaCl}$ are shown in Figs. 5a and 5b.

Fig. 5 shows that it is possible to form multilayers with these polyelectrolytes, but it is also obvious that the formation of the multilayers, i.e., the interaction between the polyelectrolytes, is largely dependant on the charge of the C-PAM. From the results in Fig. $5 \mathrm{~b}$ it can be concluded that C-PAM 2-4 give similar results, in $\mu \mathrm{eq} / \mathrm{m}^{2}$, for the build-up of PEM, whereas the build-up of PEM with C-PAM 1 shows an anomalous pattern. Obviously a certain charge density of the C-PAM is necessary in order to give a stable buildup of the PEM, and the charge density of C-PAM 1, i.e., $0.58 \mathrm{meq} / \mathrm{g}$, is too low. However, despite the leveling off in adsorption for C-PAM 1/A-PAM (compared to C-PAM24) as the number of layers increases, it is striking to notice that there is a change in adsorption upon addition of C-PAM and A-PAM, respectively, in each consecutive layer. The reason for this behavior during the formation of the multilayers is not clear, even though it is tempting to suggest a combined adsorption/desorption process as the polyelectrolytes are added to an earlier formed adsorbed layer. This process has been suggested in earlier investigations [34] where a balance between adsorbed and free polyelectrolyte complexes

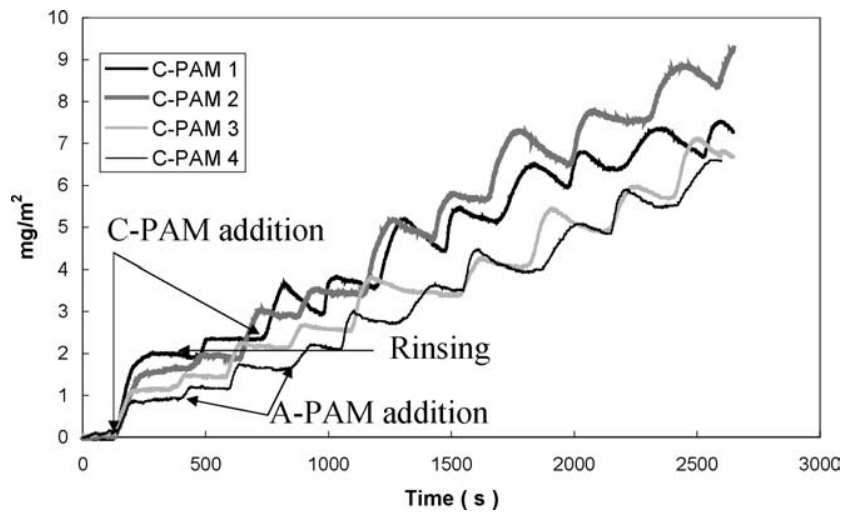

(a)

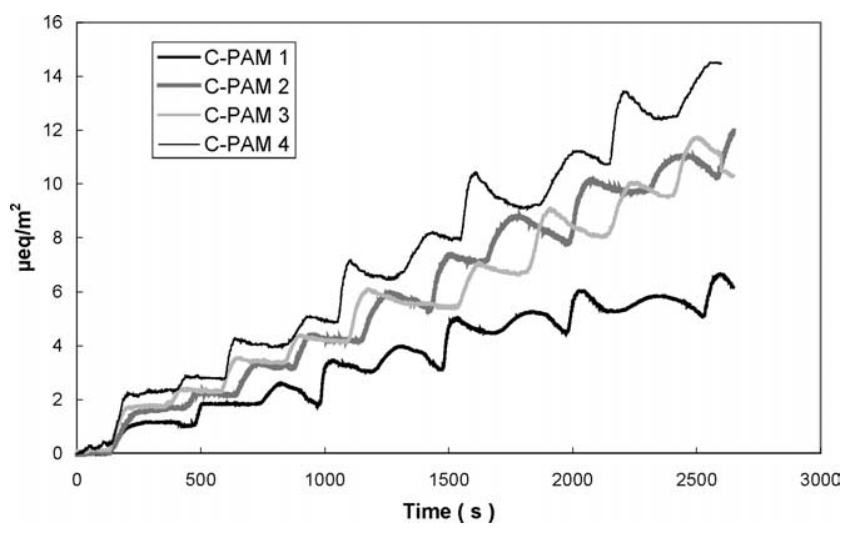

(b)

Fig. 5. Formation of multilayers of C-PAM and A-PAM on $\mathrm{SiO}_{2}$ surfaces at $\mathrm{pH} 9$ and at $0.01 \mathrm{M} \mathrm{NaCl}$. (a) Adsorbed amount in $\mathrm{mg} / \mathrm{m}^{2}$ determined from the $\Delta S / S_{0}$ values as described earlier. (b) Adsorbed amount of charges in $\mu \mathrm{eq} / \mathrm{m}^{2}$, calculated from the adsorbed amount in each layer and the charge of the polyelectrolyte according to Table 1 .

was introduced to explain the initial increase in adsorption, followed by a slow decrease in the adsorbed amount. Another explanation could be a rearrangement of the polyelectrolytes in the adsorbed layer, resulting in a change in the reflectometer signal. This will be discussed further in connection with the results presented in Fig. 6 and these results indicate that it is more a rearrangement than a desorption process that is being observed for the C-PAM 1/A-PAM polymer system.

Despite the difference in the general slopes of the curves corresponding to C-PAM 2-4 and C-PAM 1, it can nevertheless be seen in Figs. 5a and 5b that the general behavior of an initial increase followed by a decrease in the adsorbed amount can be seen for all curves from the formation of the third polyelectrolyte layer and upward. It must also be kept in mind that the adsorbed amount is calculated from the change in the optical signal, expressed as $\Delta S / S_{0}$. A change in the optical properties of the adsorbed layer can also explain the observed decrease in the signal.

To investigate whether desorption occurs upon the introduction of the polyelectrolytes in each layer, the adsorption of C-PAM 3 and A-PAM at $\mathrm{pH} 9$ and $0.01 \mathrm{M} \mathrm{NaCl}$ was investigated by using the QCM-D. The results from these 


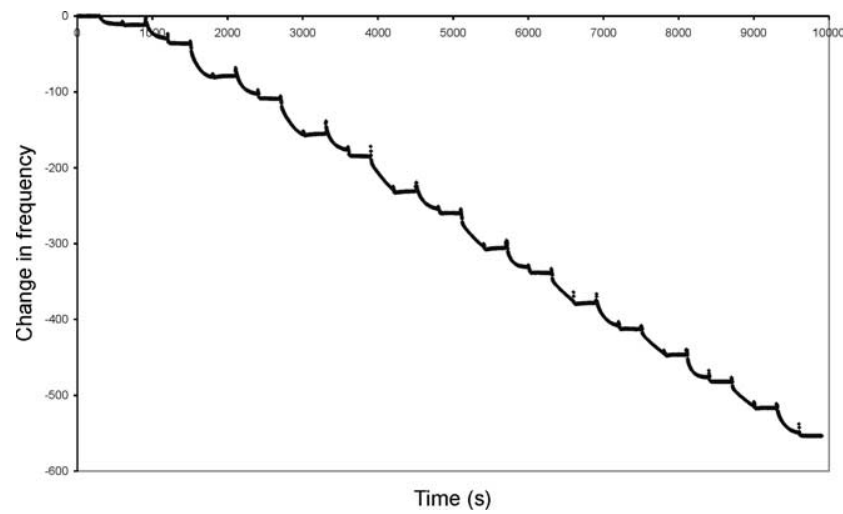

(a)

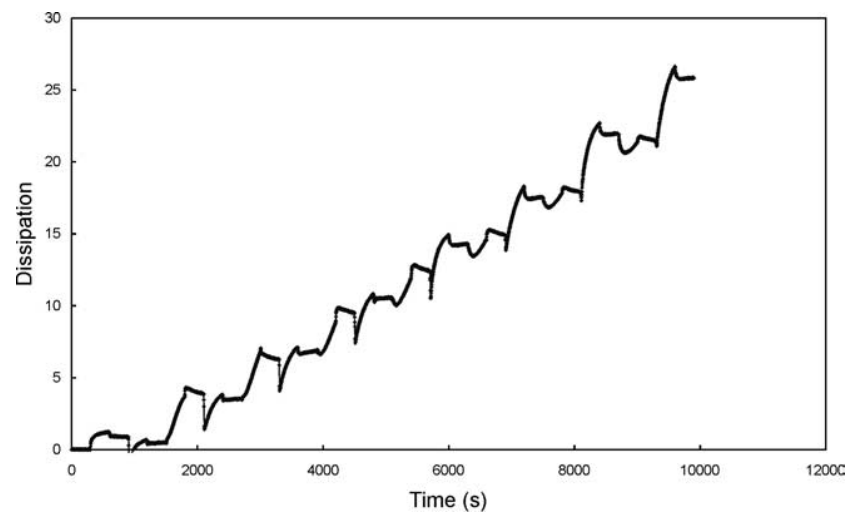

(b)

Fig. 6. Determination of the build-up of multilayers from C-PAM 3 and A-PAM on $\mathrm{SiO}_{2}$ surfaces at $\mathrm{pH} 9$ and at $0.01 \mathrm{M} \mathrm{NaCl}$ with the aid of the QCM-D equipment. (a) Change in frequency upon adsorption and rinsing; (b) change in dissipation upon adsorption and rinsing. Only data from the third overtone have been included in these results.

measurements are shown in Fig. 6. Fig. 6a shows the change in frequency as a function of adsorption. The $\Delta F$ values are proportional to adsorbed mass according to the Sauerbrey equation [35]. However, no attempt was made to convert the measured decrease in frequency to a surface excess due to the unknown contribution of trapped water within the multilayer, which is registered as a mass uptake [36]. Fig. 6b shows the energy dissipated through the multilayer as measured by QCM-D. Suspending the voltage supplied to the quartz crystal and measuring the decay of the amplitude of the crystal as a function of the time determines the dissipation. Upon adsorption of a rigidly attached species, the change in dissipation is small. The change in dissipation is high for an adsorbed layer with significant viscoelastic properties. The same procedure as for the measurements with the SPAR equipment was used during these experiments. This means that the adsorption was followed by rinsing with $0.01 \mathrm{M} \mathrm{NaCl}$ before the formation of the next polyelectrolyte layer, and so forth. It has to be pointed out that the hydrodynamic situation is not the same in the SPAR and in the QCM-D, but it is not believed that this would not invalidate the comparison between the two methods.
As shown by the results in Fig. 6a, a steady decrease in frequency is observed, i.e., an increase in adsorption, as the surface is consecutively treated with cationic and anionic polyelectrolytes, respectively. There is no sign of desorption, which would have been seen as an increase in the frequency. Naturally the frequency signal contains both the adsorbed amount of polyelectrolyte and the amount of immobilized water, but it is considered highly unlikely that a desorption of polyelectrolyte and an increase in amount of immobilized water upon desorption would result in a constant-frequency signal. The most likely interpretation of the data is that there is no desorption and that the decrease in the $\Delta S / S_{0}$ signal in the SPAR equipment after the initial increase in adsorption is the result of an optical artefact due to the rearrangement of the polyelectrolyte in the adsorbed layer following the initial adsorption. This hypothesis is also supported by the data shown in Fig. 6b, which displays the change in dissipation as polyelectrolyte is adsorbed. According to Fig. 6b, the general trends as observed from the SPAR data can be recognized also in the dissipation data. This is especially valid for the adsorption of the cationic polyelectrolyte from the third layer upward. As can be seen in Fig. 6b, an initial rise in the dissipation as the C-PAM is introduced is followed by a decrease in the dissipation, indicating a rearrangement of the polyelectrolytes within the adsorbed layer. As the anionic polyelectrolyte is introduced, there is an initial decrease in the dissipation, followed by a slow increase. This indicates that as the A-PAM is introduced into the preformed layer, there is a decrease in the mobility within the adsorbed layer, but as the adsorption continues this mobility (i.e., the viscous losses) increases again. This also indicates that the initial increase, followed by a decrease detected in the SPAR upon exposure to fresh polyelectrolyte solution, at least for C-PAM 2-4, is a result of a combination of an increase in the adsorbed amount and a change in the internal structure of the formed layer. These combined results from SPAR and QCM-D give new insights into the processes occurring in the adsorbed layer but, needless to say, more experiments are needed to investigate these suggested processes further. Other recent investigations have also shown the large potential of using combinations of QCM and ellipsometric data and this has been demonstrated earlier [36]. It should also be added that the large deviation of the C-PAM 1 data from the C-PAM 2-4 suggests that some desorption might occur for this type of polymer.

Having established that the desorption is not necessarily the dominant mechanism for the detected decrease in the adsorption data from the SPAR, it can safely be assumed that the initial increase in the $S$-value upon polymer adsorption is due to binding of the polyelectrolyte to the surface. This $\Delta S$-value, as schematically defined in Fig. 7, was then used to calculate the number of adsorbed charges in each consecutive layer. It should be stressed that this way of treating the data is based on the assumption that the decrease in the $\Delta S$ value after the maximum is due to a restructuring of the adsorbed layer and not to desorption of polyelectrolyte. 


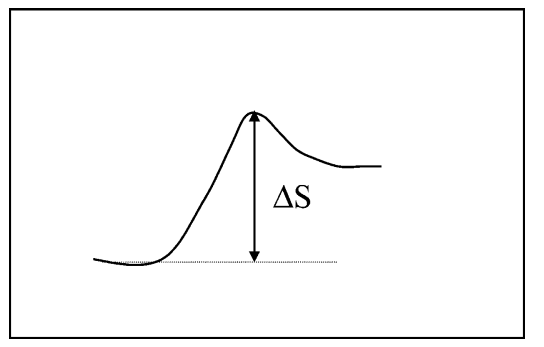

Fig. 7. Schematic description of how the $\Delta S$ value for each layer was determined from the reflectometry data.

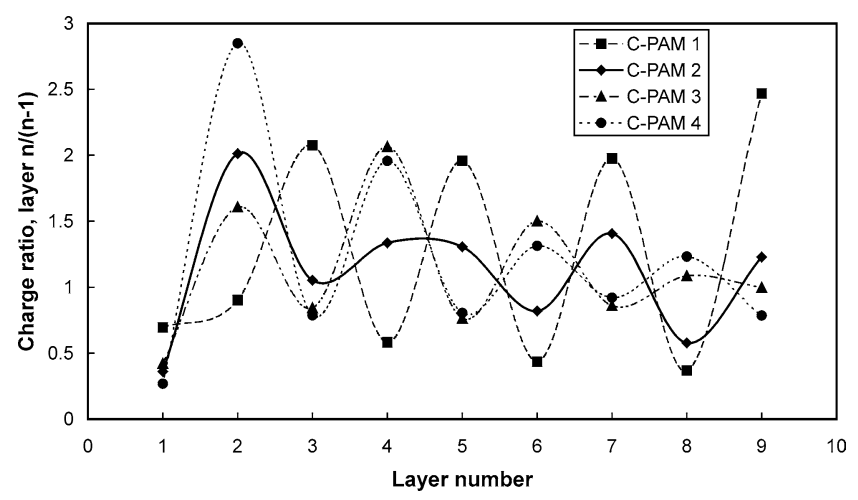

Fig. 8. Charge ratio between the numbers of polyelectrolyte charges adsorbed in adjacent layers on the $\mathrm{SiO}_{2}$ surface, as estimated from the SPAR measurements. The experiments were conducted at $\mathrm{pH} 9$ and at $0.01 \mathrm{M}$ $\mathrm{NaCl}$.

This assumption is based on the QCM measurements and suggests that the refractive index of the adsorbed layer is changed in a nonlinear way due to the interaction between the two types of polyelectrolytes. This hypothesis definitely needs to be tested in future investigations. However, the ratio of adsorbed charges in two adjacent layers, calculated based on the assumptions shown in Fig. 7, could then be calculated from the charge of the respective polyelectrolyte.

The results from these calculations are shown in Fig. 8 for the adsorption of C-PAM 1-4 and A-PAM at $\mathrm{pH} 9$ and at a salt concentration of $0.01 \mathrm{M} \mathrm{NaCl}$. As can be seen in Fig. 8, the surface will influence the adsorption of the polyelectrolytes up to about five layers from the surface. After five layers, the adsorption is totally dominated by the interaction between the polyelectrolyte; i.e., there is a 1:1 stoichiometry between the charges on the polyelectrolytes in adjacent layers. This result is also in accordance with earlier measurements for other types of polyelectrolytes [12,19]. As earlier discussed, the C-PAM with the lowest charge shows a large deviation from this general behavior and no clear 1:1 stoichiometry between charges in adjacent layers is found for this polyelectrolyte combination.

The results regarding the build-up of the PEM so far have been limited to $\mathrm{pH} 9$ and $0.01 \mathrm{M} \mathrm{NaCl}$ and it was therefore considered important to investigate the build-up of PEM at $\mathrm{pH} 6$ and $0.01 \mathrm{M} \mathrm{NaCl}$ in order to clarify if there is any large influence of the surface charge on the PEM formation.

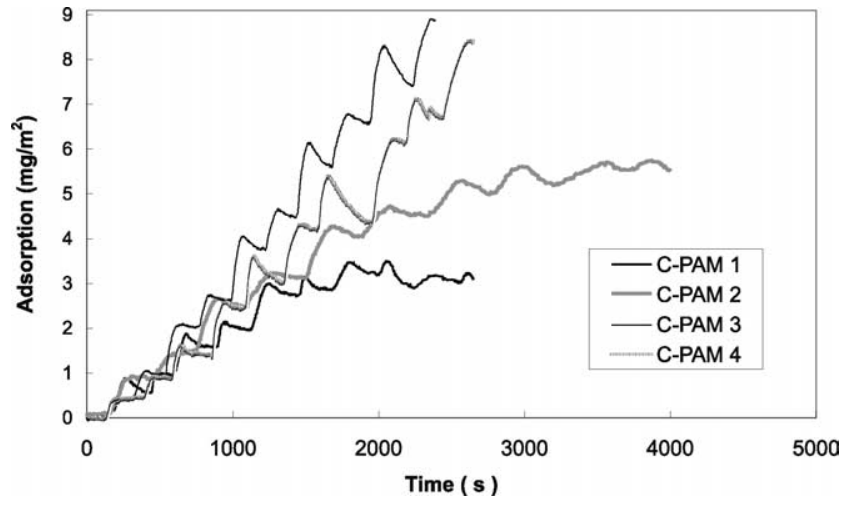

Fig. 9. Formation of multilayers of C-PAM and A-PAM on $\mathrm{SiO}_{2}$ surfaces at $\mathrm{pH} 6$ and $0.01 \mathrm{M} \mathrm{NaCl}$. The adsorbed amount in $\mathrm{mg} / \mathrm{m}^{2}$ has been determined from the $S / S_{0}$ values as described in Section 2.

Results from these measurements are shown in Fig. 9, where the adsorption in $\mathrm{mg} / \mathrm{m}^{2}$ is shown.

It is clear from these results that the general trends are the same as those detected in Fig. 5 for adsorption at $\mathrm{pH}$ 9. Despite the fairly large difference in the adsorption in the first layers, as shown in Figs. 1-3, the adsorption for C-PAM 3 and C-PAM 4 is almost the same for layers of higher order at $\mathrm{pH} 6$ and at $\mathrm{pH}$ 9. This again demonstrates that the surface has the greatest influence on the first layers but that the interaction between the polyelectrolytes will dominate the adsorption for layers of higher order and hence even out the initial difference in adsorption. It is also obvious that there is a deviation not only for C-PAM 1 but also for CPAM 2 from the trend of a linear build-up of the adsorbed amount with increasing layer number. This indicates that a certain critical interaction energy between the surface and the polyelectrolyte is needed in order to construct stable PEMs. Furthermore the strong deviation from 1:1 stoichiometry between the polyelectrolytes in adjacent layers for the C-PAM with the lowest charge density also demonstrates that this polyelectrolyte combination is close to the limit of a critical interaction energy for multilayer formation. The factors controlling this critical interaction energy are not known and further development of the theories for PEM formation is needed to identify these contributing factors.

\section{Conclusions}

The results in the present report have shown that bilayers and multilayers of cationic and anionic polyacrylamide are formed on $\mathrm{SiO}_{2}$ surfaces both in deionized water and in $0.01 \mathrm{M} \mathrm{NaCl}$. It was also shown that the charge of the cationic polyacrylamide has a significant influence on the build-up of these layers. The adsorption of cationic polyacrylamide in the first layer is highly dependent on the $\mathrm{pH}$ at which the adsorption experiments are performed. At $\mathrm{pH} 6$ the adsorption is controlled both by electrostatic and nonionic interactions, whereas the adsorption at $\mathrm{pH} 9$ is also controlled by geometrical restrictions; i.e., the surface is to- 
tally covered by C-PAM molecules before all the charges have been neutralized. This means that the $\mathrm{SiO}_{2}$ surface can be recharged by the C-PAM both at $\mathrm{pH} 6$ and at $\mathrm{pH} 9$, but the controlling factors for this recharging are different.

The adsorption of A-PAM in the second layer is directly related to the adsorption stoichiometry between the polyelectrolyte and the surface in the first layer when the adsorption is performed at $\mathrm{pH} 9$ in deionized water and in $0.01 \mathrm{M}$ $\mathrm{NaCl}$. At pH 6 this relationship is not as clear for the adsorption from deionized water. The addition of $\mathrm{NaCl}$ has a significant effect on the amount of A-PAM that can be adsorbed in the second layer at $\mathrm{pH}$ 6. This is most likely due to the change in adsorbed layer structure as a function of increased added electrolyte.

The formation of multilayers of C-PAM with different charge densities and A-PAM is very similar, except for the C-PAM with the lowest charge density. It is also found that the charge ratio between polyelectrolytes in adjacent layers reaches a value of 1 when more than five layers are adsorbed onto the surface for all polymers, except for the C-PAM with the lowest charge density.

The data from the reflectometer show an initial increase followed by a significant decrease as every layer is formed for layers of higher order, i.e., larger than 5. Comparison between reflectometer data and data from QCM-D measurements strongly indicated that this behavior was not an effect of an actual decrease in surface excess, but more an effect of a change in the ordering of polyelectrolytes in the layers during the adsorption process.

Furthermore, the results also showed that the critical charge of the polyelectrolyte for the formation of multilayers was also dependent on the charge of the substrate; i.e., the lower the surface charge the higher the critical charge of the C-PAM necessary for the formation of a multilayer.Finally, the combination of QCM and reflectometer/ellipsometer data also shows great potential for future investigations regarding the structure and amount of polyelectrolyte in adsorbed layers.

\section{References}

[1] G.J. Fleer, M.A. Cohen Stuart, J.M.H.M. Scheutjens, T. Cosgrove, B. Vincent (Eds.), Polymers at Interfaces, Chapman \& Hall, London, 1993, pp. 343-375.

[2] M. Muthukumar, J. Chem. Phys. 86 (1987) 7230.

[3] A.V. Dobrynin, A. Deshovski, M. Rubinstein, Macromolecules 34 (2001) 3421-3436.
[4] J.-F. Joanny, Eur. Phys. J. B 9 (1999) 117.

[5] R.R. Netz, J.-F. Joanny, Macromolecules 32 (1999) 9013.

[6] B. Dobiáš (Ed.), Coagulation and Flocculation-Theory and Applications, in: Surfactant Sci. Series, vol. 47, Dekker, New York/Basel/ Hong Kong, 1993.

[7] T.F. Tadros (Ed.), The Effect of Polymers on Dispersion Properties, Academic Press, London, 1982.

[8] G. Decher, Science 277 (1997) 1232.

[9] M.A. Cohen Stuart, N. Hoogeveen, G.J. Fleer, M. Böhmer, Langmuir 12 (1996) 3675.

[10] P. Bertrand, A. Jonas, A. Laschewsky, R. Legras, Macromol. Rapid Commun. 21 (2000) 319-348.

[11] S.K. Tripathy, J. Kumar, H.S. Nalwa (Eds.), Handbook of Polyelectrolytes and Their Application. 1. Polyelectrolyte-Based Multilayers, Self-Assemblies and Nanostructures, Am. Sci. Publishers, New York, 2002.

[12] G. Decher, J.B. Schlenoff (Eds.), Multilayer Thin Films, Wiley/VCH, New York/Weinheim, 2002.

[13] P. Linse, V. Schubin, J. Phys. Chem. 99 (1995) 1285.

[14] P. Linse, Schubin, Macromolecules 30 (1997) 5944.

[15] M.A. Cohen Stuart, C.W. Hoogendam, A. de Kaizer, J. Phys. Condensed Matter 9 (1997) 7767.

[16] C. Geffroy, M.P. Labeau, K. Wong, B. Cabane, M.A. Cohen Stuart, Colloids Surf. A Physicochem. Eng. Aspects 172 (2000) 47.

[17] S.Y. Park, M.F. Rubner, A.M. Mayes, Langmuir 18 (2002) 9600.

[18] C. Picart, J. Mutterer, L. Richert, Y. Luo, G.D. Prestwich, P. Schaaf, J.-C. Voegel, P. Lavalle, Proc. Natl. Acad. Sci. USA 99 (20) (2002) 12531.

[19] J.B. Schlenoff, H. Ly, M. Li, J. Am. Chem. Soc. 120 (1998) 7626.

[20] N. Kato, P. Schuetz, A. Fery, F. Caruso, Macromolecules 35 (2002) 9780.

[21] G. Decher, in: G. Decher, J.B. Schlenoff (Eds.), Multilayer Thin Films, Wiley/VCH, New York/Weinheim, 2002, p. 17.

[22] R. Aksberg, L. Ödberg, Nordic Pulp Paper Res. J. 5 (1990) 168.

[23] J.C. Dijt, M.A. Cohen-Stuart, J.E. Hofman, G.J. Fleer, Colloids Surf. 51 (1990) 141.

[24] N.G. Hoogeveen, M.A. Cohen-Stuart, G.J. Fleer, J. Colloid Interface Sci. 182 (1996) 146

[25] L. Wågberg, I. Nygren, Colloids Surf. A Physicochem. Eng. Aspects 159 (1999) 3.

[26] H. Terayama, J. Polym. Sci. 8 (1952) 243.

[27] D. Horn, Progr. Colloid Polym. Sci. 65 (1978) 251.

[28] F. Mabiré, R. Audebert, C. Quivoron, Polymer 25 (1984) 1317.

[29] W.-M. Kulicke, R. Kniewske, Macromol. Chem. 182 (1988) 2277.

[30] L. Wågberg, D. Solberg, Colloids Surf. A Physicochem. Eng. Aspects (2003), in press.

[31] R.H. Iler, The Chemistry of Silica, Wiley, New York, 1979, pp. 356, 661.

[32] V. Schubin, J. Colloid Interface Sci. 191 (1997) 372.

[33] F. Mabiré, R. Audebert, C. Quivoron, Polymer 25 (1984) 1317.

[34] D. Kovacevic, S. van der Burgh, A. de Kaizer, M.A. Cohen Stuart, Langmuir 18 (2002) 5607.

[35] G. Sauerbrey, Z. Phys. 155 (1959) 206.

[36] F. Höök, B. Kasemo, C. Fant, K. Sott, H. Elwing, Anal. Chem. 73 (2001) 5796. 